\title{
A neural transplantation cannula and microinjector system: experimental and clinical experience
}

\section{Technical note}

\section{Ivar Mendez, M.D., Ph.D., Murray Hong, Ph.D., Stephen Smith, P. Eng., Alain Dagher, M.D., and Jacques Desrosiers, M.D.}

Department of Surgery, Division of Neurosurgery, Dalhousie University and Queen Elizabeth II Health Sciences Centre, Halifax, Nova Scotia, Canada; Biomedical Engineering Department, IWK-Grace Health Centre, Halifax, Nova Scotia, Canada; and McConnell Brain Imaging Centre, Montreal Neurological Institute, McGill University, Montreal, Quebec, Canada

The authors present a simple, reliable, and safe system for performing neural transplantation in the human brain. The device consists of a transplantation cannula and microinjector system that has been specifically designed to reduce implantation-related trauma and to maximize the number of graft deposits for each injection. The system was evaluated first in an experimental rat model of Parkinson's disease (PD). Animal transplantation with this system showed excellent graft survival with minimal trauma to the brain. Following this experimental stage, the cannula and microinjector system was used in eight patients with PD enrolled in the Halifax Neural Transplantation Program who received bilateral putaminal transplants of fetal ventral mesencephalic tissue. A total of 16 transplantation operations and 64 trajectories were performed in the eight patients, and there were no intra- or perioperative complications. Magnetic resonance imaging studies obtained 24 hours after surgery revealed no evidence of tissue damage or hemorrhage. Transplant survival was confirmed on fluorodopa positron emission tomography scans 6 and 12 months after surgery.

As neural transplantation procedures for the treatment of neurological conditions evolve, the ability to deliver viable grafts safely will become of critical importance. The device presented here has been proven to be of value in maximizing the number of graft deposits while minimizing implantation-related trauma to the host brain.

Key Words * neural transplantation * stereotactic cannula * Parkinson's disease * dopaminergic graft

Neural transplantation of fetal ventral mesencephalic (VM) tissue has been studied for the past two decades as a potential surgical strategy for the treatment of Parkinson's disease (PD). Clinical trials in 
patients with PD have been conducted in several centers worldwide. To date more than 200 patients have received fetal tissue into the striatum.[11,17,18,22] Survival of the grafts has been documented using positron emission tomography (PET) scanning[6,19,23] as well as postmortem studies.[10] Although the results of these trials have been promising, [7,23] clinical efficacy has not reached the stage at which neural transplantation could become a routine therapeutic procedure for patients with PD.

Implantation-related trauma, which decreases graft survival, and inadequate reinnervation of the host striatum due to suboptimal distribution of graft deposits are considered detrimental factors in achieving optimum clinical efficacy. Minimized implantation-related trauma and a more complete reinnervation of the dopamine-depleted striatum have been achieved in animal models of PD by decreasing the size of the implantation cannula and increasing the number of deposits of fetal dopaminergic cells.[14-16] These modifications to the implantation technique have produced improvements in host reinnervation and functional recovery in the rodent model of PD.[14-16]

We report here the experimental and clinical experience with the use of a neural transplantation cannula and microinjector system that has been specifically designed to decrease implantation-related trauma and to maximize the number of graft deposits per injection.

We conducted studies using the rat model of PD in which excellent graft survival with minimal trauma to the brain was demonstrated. Following this experimental stage, the cannula and microinjector system were used in eight patients with PD enrolled in the Halifax Neural Transplantation Program who received bilateral putaminal transplants of fetal VM tissue.

\section{LABORATORY AND CLINICAL MATERIALS AND METHODS}

\section{Implantation Cannula and Microinjector System}

The cannula and microinjector system (Fig. 1 left) were designed to fit a 50- $\mu$ l Hamilton syringe and can be adapted to be used with any stereotactic frame system. We used the Leksell stereotactic frame (Fig. 1 right), and the only modification needed was to change the stop and guide portions to a custom-made set with the appropriate diameter $(0.8 \mathrm{~mm})$ for the cannula. The cannula was manufactured from a stainless steel 21-gauge needle (outer diameter $0.8 \mathrm{~mm}$ ) with a length of $195 \mathrm{~mm}$. A standard Luer lock was fitted to the proximal end. The cannula tip was rounded and polished to minimize trauma. The proximal end has two side holes $(0.3-\mathrm{mm}$ diameter). The first hole is located $1 \mathrm{~mm}$ proximal to the tip and the second hole is $2 \mathrm{~mm}$ proximal to the first hole but oriented in the opposite direction (Fig. 1 left). 


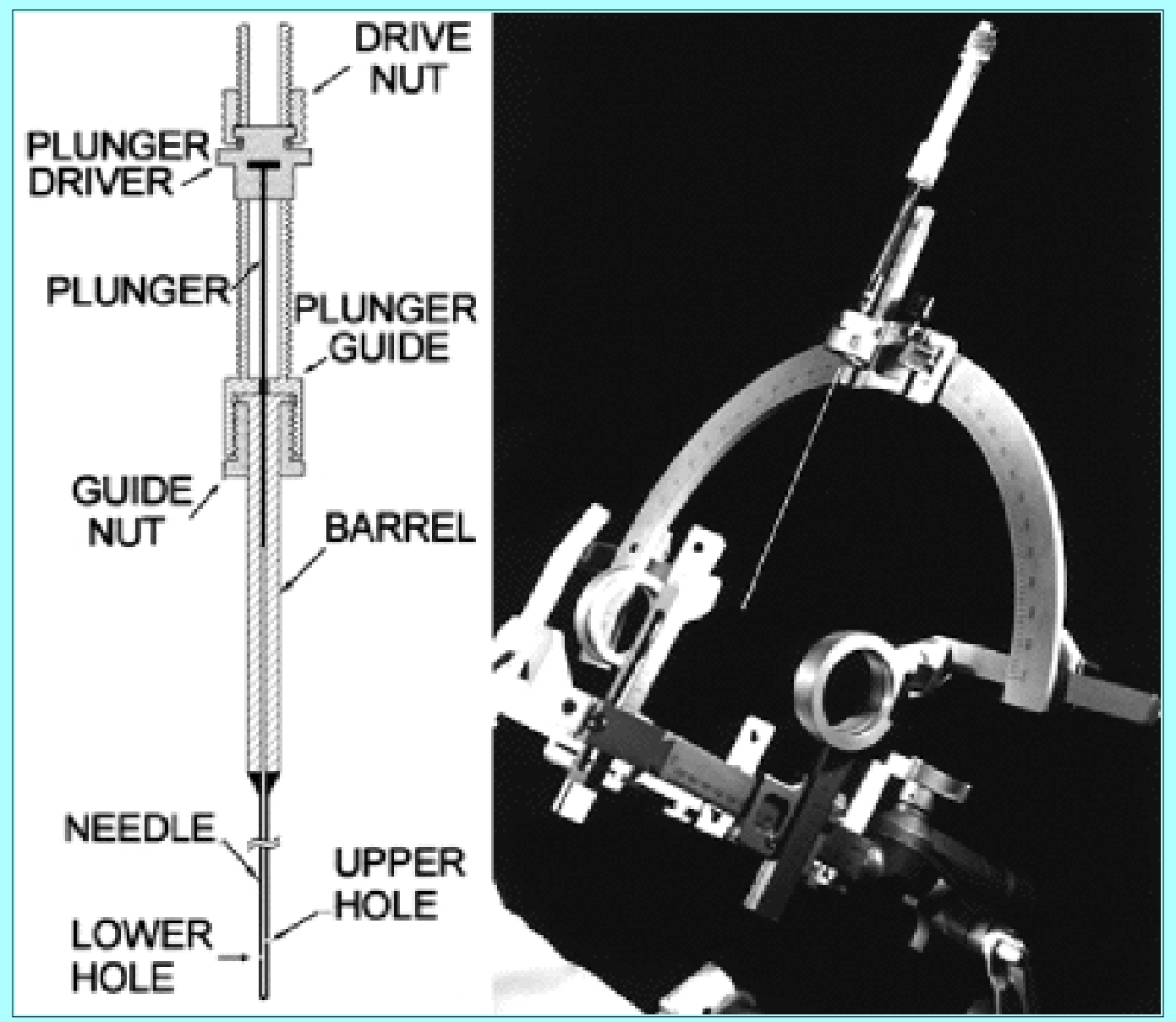

Fig. 1. Left: Diagram showing a 50- $\mu$ l Hamilton syringe fitted with the microinjector system and the neural transplantation cannula. Note the orientation of the side holes on opposite sides of the cannula tip. Right: Photograph showing the neural transplantation cannula and microinjector system fitted to the Leksell stereotactic frame.

The microinjector system was manufactured of acetal nylon and ionized aluminum. The microinjector consists of a threaded cylinder with an adapter for the syringe barrel placed distally and a plunger driver proximally (Fig. 1 left). The syringe plunger is controlled by the plunger driver and can deliver the desired volume of cell suspension accurately.

\section{Animal Experiments}

Ten female Wistar rats weighing 200 to $225 \mathrm{~g}$ (housed two animals per cage) were given free access to food and water and were allowed to acclimatize to their surroundings in the animal-care facility for 7 days prior to surgery or behavioural testing. All animal procedures were performed in accordance with the guidelines of the Canadian Council on Animal Care and of the University Council on Laboratory Animals. After induction of pentobarbital anesthesia, rats received two stereotactic injections of 6-hydroxydopamine (6-OHDA) into the right ascending mesostriatal dopaminergic pathway at the following coordinates: 1) $2.5 \mu \mathrm{l}$ of 6-OHDA (3.6 $\mu \mathrm{g}$ 6-OHDA hydrobromic acid/ $\mu \mathrm{l}$ in $0.2 \mu \mathrm{g} / \mu \mathrm{l}$ L-ascorbate $0.9 \%$ saline) anteroposterior -4.4 , L 1.2, dorsoventral -7.8 , tooth bar -2.4 and 2) $3 \mu \mathrm{l}$ of $6-O H D A$ at anteroposterior -4.0 , lateral 0.8 , DV -8.0 , tooth bar +3.4 . The injection rate was approximately $1 \mu \mathrm{l} /$ minute, and the cannula was left in place for an additional 4 minutes before being slowly retracted. Following a 2-week recovery period in the animal-care facility, the rats were given an amphetamine challenge $(5 \mathrm{mg} / \mathrm{kg}$ intraperitoneally) and their rotational scores were collected over a 90-minute period. Only animals exhibiting a mean ipsilateral rotation score of 9 or higher of full body 
turns/minute were included in the study.

The lesioned rats underwent transplantation surgery in which the clinical cannula and microinjector system was used. In brief, cell suspensions were prepared from the VM of 14-day-old rat fetuses and injected stereotactically into the host brains of 6-OHDA-treated animals. Cell suspensions of the fetal VM tissue were prepared by the following procedure. The tissue was incubated in $0.1 \%$ trypsin/ $0.05 \%$ DNase/Dulbecco's modified eagle medium at $37^{\circ} \mathrm{C}$ for 20 minutes and then rinsed four times in $0.05 \%$ DNase/Dulbecco's modified eagle medium. After this it was mechanically dissociated into a "chunky" cell suspension. A final cell concentration of approximately 200,000 cells/ $\mu$ l was used with viability of $98 \%$ as determined by the trypan blue dye exclusion method. A total of 500,000 cells $(2.5 \mu \mathrm{l})$ were implanted into the dopamine-depleted striatum.

Six to 8 weeks after transplantation, the rats were killed using an overdose of pentobarbital and perfused transcardially with $100 \mathrm{ml}$ of $0.1 \mathrm{M}$ phosphate buffer, which was followed by $250 \mathrm{ml}$ of $4 \%$ freshly depolymerized paraformaldehyde in $0.1 \mathrm{M}$ phosphate buffer over 15 minutes. The brains were removed, postfixed overnight in $4 \%$ paraformaldehyde in $0.1 \mathrm{M}$ phosphate buffer, and stored in phosphate-buffered saline containing $30 \%$ sucrose for 24 hours. Coronal sections $40-\mu \mathrm{m}$ thick were cut on a freezing microtome and collected serially in $0.1 \mathrm{M}$ phosphate buffer. Sections were processed for tyrosine hydroxylase $(\mathrm{TH})$ immunohistochemical analysis by using a primary rabbit anti-TH antiserum (1:2500 Pel Freeze) and the ABC kit.

\section{CLINICAL TRIALS}

Eight patients enrolled in the Halifax Neural Transplantation Program received bilateral putaminal fetal VM tissue obtained from women undergoing elective abortions in the pregnancy-termination unit of our center. Strict guidelines of a protocol approved by the university and hospital ethical review boards were followed.

The surgical transplantation procedures were performed in two stages in each side 4 to 6 weeks apart. Patients were admitted to the hospital the night prior to surgery. On the day of surgery patients were fitted with a Leksell stereotactic head frame after the induction of a local anesthetic. The stereotactic coordinates for targets in the putamen were calculated using $\mathrm{T}_{1}$-weighted magnetic resonance (MR) images and a computerized planning workstation. The patient received local anesthesia and sedation, and transplantation was performed using a combination of midazolam $(0.25-1.0-\mathrm{mg}$ bolus doses) and propofol (10-20-mg bolus followed by infusion at $15-40 \mu \mathrm{g} / \mathrm{kg} / \mathrm{minute})$. A burr hole was made at the level of the coronal suture, and the transplantation cannula was inserted into four different trajectories approximately $3 \mathrm{~mm}$ apart in the postcommissural putamen. A 50-ml Hamilton syringe, fitted with the microinjector, was used to load the $15 \mu \mathrm{l}$ of cell suspension in the transplantation cannula. The cell suspension was prepared in the same manner as described in Animal Experiments. The dead space in the cannula was filled first with medium solution in such a way that the $15 \mu$ l of cell suspension was only loaded in the most distal part of the cannula. The cell suspension was deposited along each of four trajectories previously calculated on the patient's MR image scan. Four injections of approximately $2.5 \mu \mathrm{l}$ each ( 8 deposits) were made in each trajectory for a total of $10 \mu \mathrm{l}$ per trajectory. The injections were made $3 \mathrm{~mm}$ apart at a rate of approximately $1 \mu \mathrm{l} /$ minute as the cannula was slowly withdrawn in a stepwise fashion and rotated $90^{\circ}$ clockwise before each injection (Fig. 2). The interval between each injection was 2 minutes and the cannula was completely withdrawn 4 minutes after the last injection. 


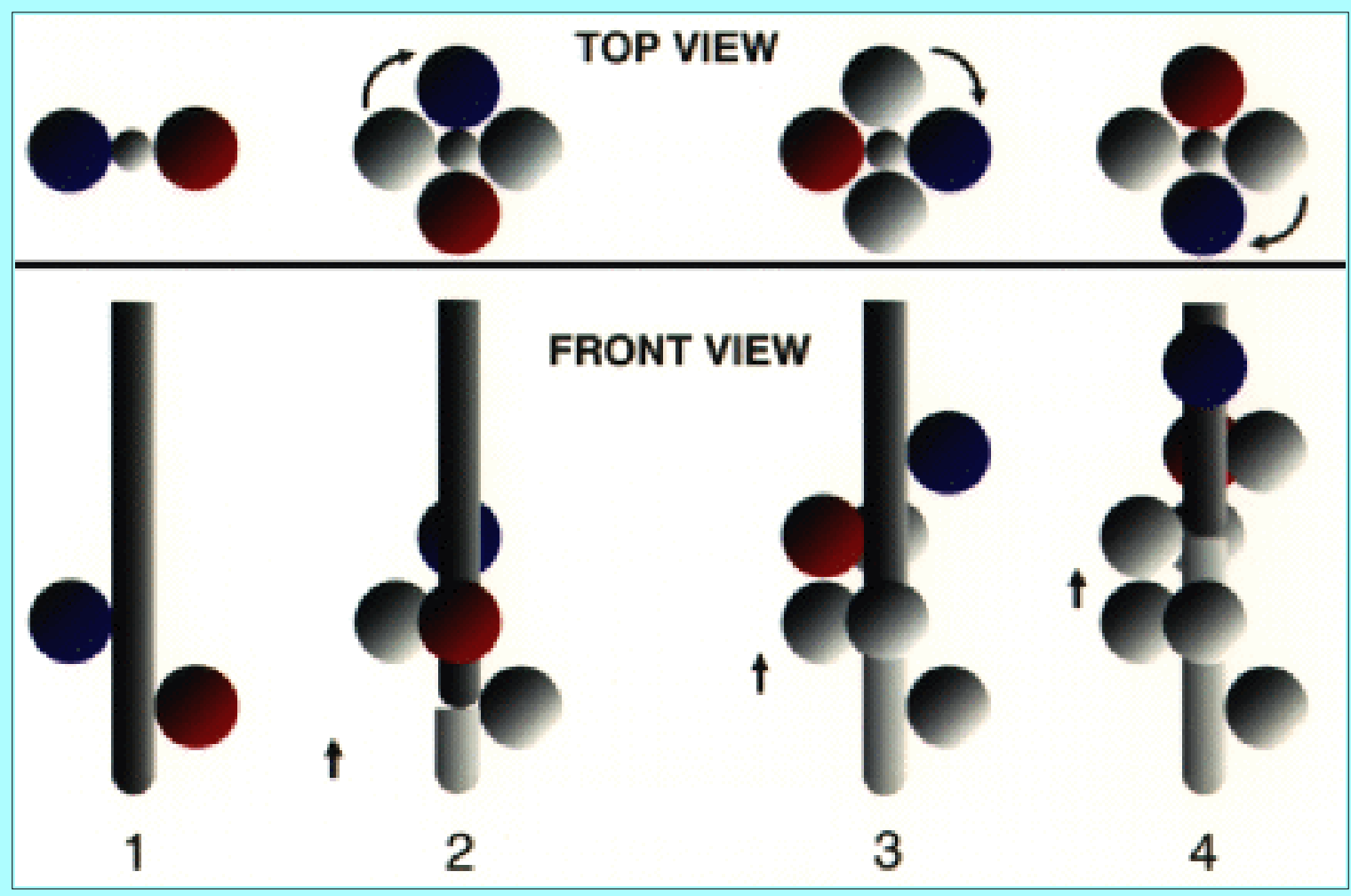

Fig. 2. Diagram showing the sequence of graft deposits by using the neural transplantation cannula (top and front views). A total of four injections are made $3 \mathrm{~mm}$ apart per trajectory. The first injection (Step 1) consists of two graft deposits oriented opposite each other (blue and red balls). The cannula is then withdrawn $3 \mathrm{~mm}$ in a stepwise fashion and rotated $90^{\circ}$ clockwise (top view), and another two deposits are made (Step 2). The process is repeated two more times (Steps 3 and 4) until a total of eight deposits per trajectory have been made (Step 4).

Approximately 4 million cells were deposited in each postcommissural putamen. Patients received $1 \mathrm{~g}$ of intravenously administered Ancef before the skin incision was made and three additional $1 \mathrm{~g}$ doses of Ancef every 8 hours postoperatively. Patients were discharged from the hospital 48 hours after surgery. Twenty-four hours postsurgery patients underwent MR imaging studies that included $\mathrm{T}_{1^{-}}$and $\mathrm{T}_{2}$-weighted as well as inversion-recovery (TR $7000 \mathrm{msec}$, TE $60 \mathrm{msec}$, and interval time $400 \mathrm{msec}$ ) sequences in the axial, coronal, and sagittal planes to assess target accuracy and bleeding. Gadolinium-enhanced MR imaging was also performed at 6 and 12 months after surgery to check for blood-brain barrier breakdown.

Positron emission tomography scanning was performed at the McConnell Brain Imaging Centre (Montreal Neurological Institute, McGill University) before and after the transplant procedure. The PET scans were obtained in three-dimensional mode, with a resolution of $5 \mathrm{~mm}$ full width at half maximum in all directions at the center of the field of view. Subjects received $5 \mathrm{mCi}$ of $[18 \mathrm{~F}]$ dihydroxyphenalamine fluorodeoxyglucose (FD) as a bolus injection into the antecubital vein over 2 minutes. Their heads were immobilized within the aperture of the PET scanner by a form-fitting vacuum device. One hour prior to scanning, the patients received carbidopa (150 mg orally) to prevent the peripheral breakdown of FD. On the day of scanning, patients did not receive antiparkinsonian medications, and they did not eat breakfast before the scanning proceedure. After the injection of FD, PET data were acquired for 90 minutes in 27 time frames of varying durations. 


\section{Sources of Supplies and Equipment}

The Wistar rats were aquired from the Charles River (St. Constant, Quebec, Canada). In the animal experiements tissue samples were incubated in trypsin obtained from Worthington (Freehold, NJ) and RNase obtained from Sigma Chemical Co. (Chicago, IL). The rabbit anit-TH antiserum was acquired from Pel Freeze Biologicals (Rogers, AR).

Both the Leksell stereotactic frame (model A0260-02), and the computerized planning workstation (Surgiplan) are produced by Elekta AB (Stockholm, Sweden). The PET scanning was performed using a tomograph (ECAT HR+ Positron Emission Tomograph) manufactured by Siemens AG (Munich, Germany).

\section{RESULTS}

\section{Animal Studies}

Animals tolerated the transplant procedure well, and all of them had grafts that survived 6 to 8 weeks posttransplantation. Typically, two graft deposits were observed in the implanted striatum, and each graft deposit corresponded to the upper- and lower-side holes of the transplant cannula (Fig. 3 upper). The cannula tract was clearly visible, connecting the upper and lower graft, and the deposits appeared to be oriented in opposite directions. Numerous TH immunoreactive cells and fibers were observed in the graft deposits and cannula tract (Fig. 3 lower). Fibers penetrating the host striatum for variable distances were also observed, and overall the appearance of the grafts was comparable to those in animals in which a glass microcapillary graft was used for grafting in our laboratory.[12,13] There was no evidence of significant trauma in the grafted area, and no tissue disruption was observed in the cannula tract. 


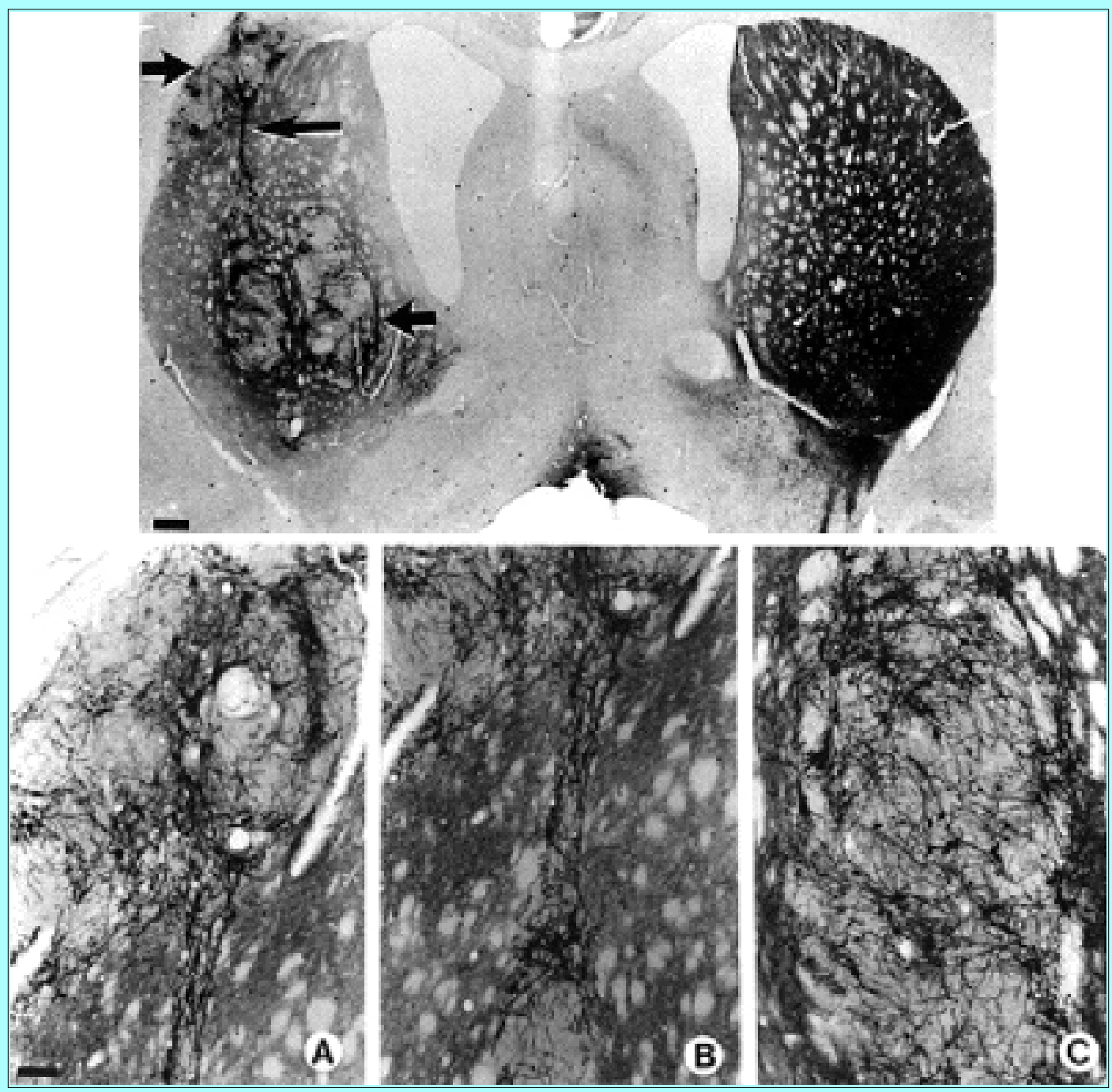

Fig. 3. Upper: Photomicrograph of a coronal section of a rat striatum immunostained with TH to visualize dopaminergic neurons. The lesioned striatum (at left) shows the two graft deposits made by the transplant cannula. Note that the orientation of the upper and lower grafts (short arrows) corresponds to the side holes of the cannula. There is no evidence of significant trauma in the transplanted striatum, and the grafts are well integrated in the host. The two grafts are connected by the cannula tract which contain TH-positive cells and fibers (long arrow). Scale bar $300 \mu \mathrm{m}$.Lower: High-power photomicrographs of the same section. A: Upper-hole graft deposit showing numerous dopaminergic cells and fibers. B: Cannula tract containing grafted cells and fibers connecting the upper and lower grafts. C: Lower-hole graft deposit. Scale bar $100 \mu \mathrm{m}$.

\section{Clinical Studies}

A total of 16 transplant operations and 64 trajectories were performed in eight patients using the implantation cannula and microinjector system. The patients tolerated the surgical procedures well, and there were no intra- or perioperative complications. The MR images of the brain obtained 24 hours after surgery revealed that the deposits were made in the desired targets in all cases (Fig. 4), and there was no evidence of hemorrhage or tissue damage. The gadolinium-enhanced MR images obtained at 6 and 12 months posttransplantation did not demonstrate any areas of enhancement, which indicates that there was no breakdown of the blood-brain barrier. 


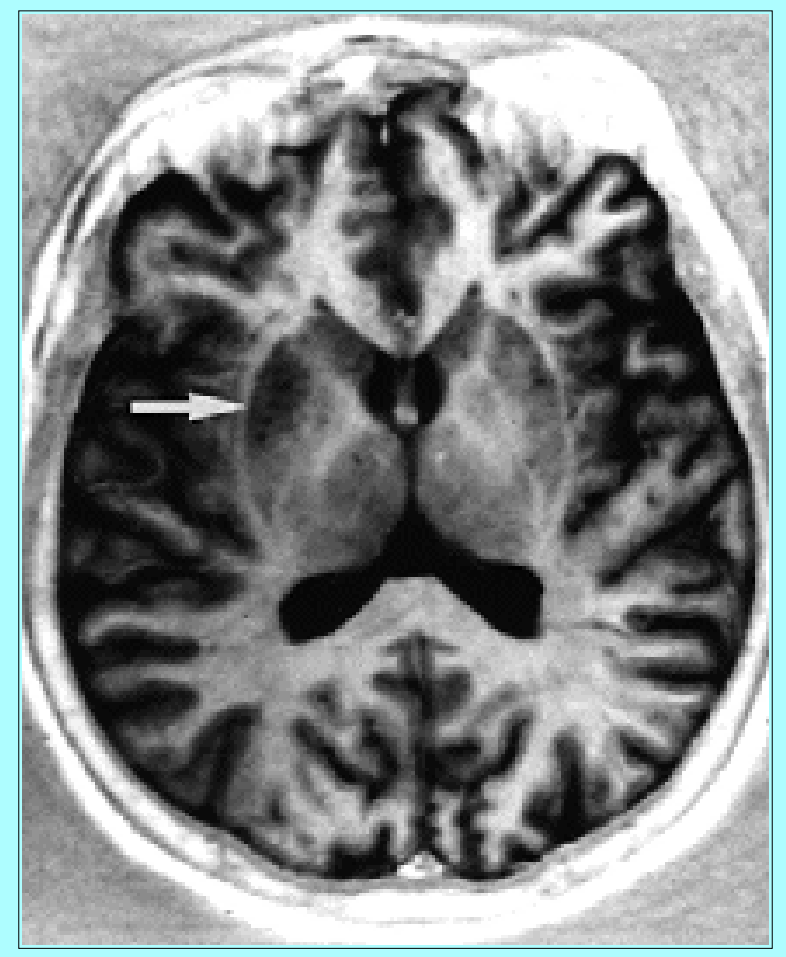

Fig. 4. Inversion-recovery MR image obtained 24 hours after surgery, demonstrating four graft deposits in the right putamen (arrow).

Postoperative fluorodopa PET scans revealed an increase in fluorodopa uptake in the transplanted areas 6 and 12 months after transplantation (Fig. 5). This increase in tracer uptake is an indication of graft survival.

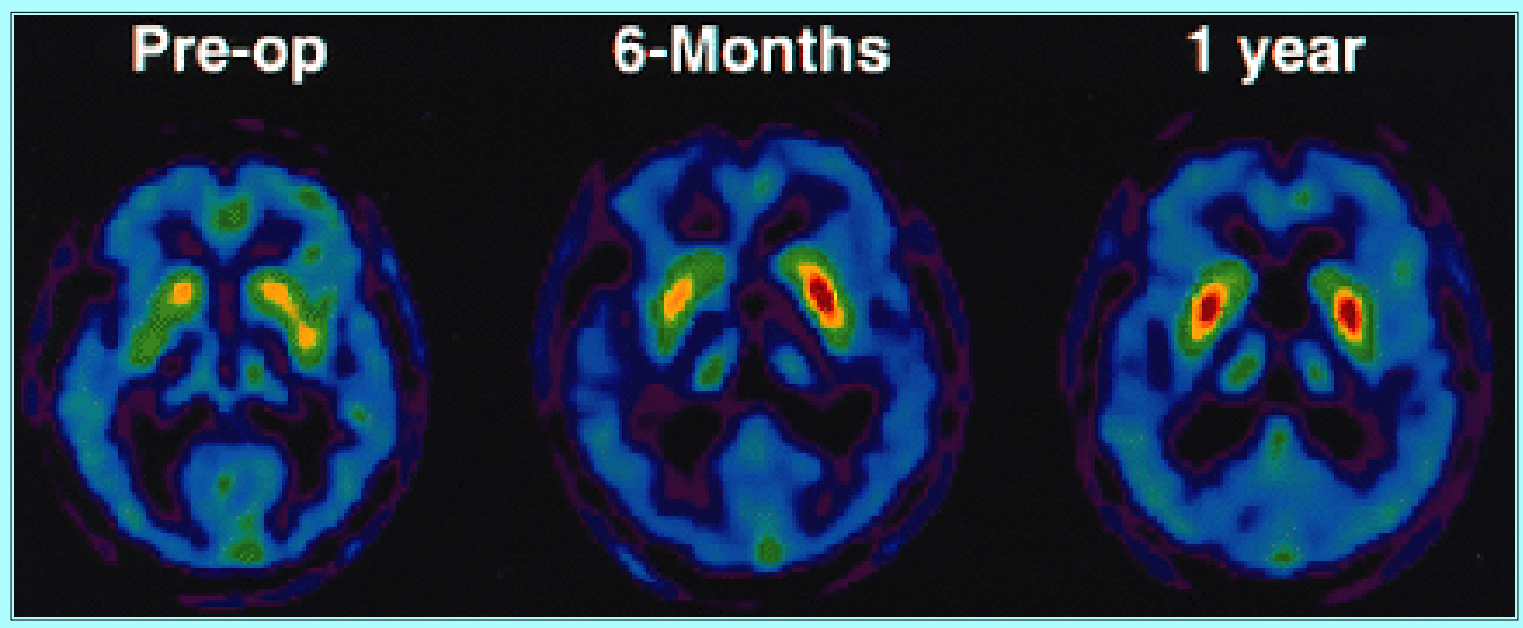

Fig. 5. Fluorodopa PET scans obtained in a patient in whom transplantation was performed using the microinjector and transplantation cannula. Sequential images from the same anatomical level obtained before the surgery and then 6 months and 1 year after bilateral putaminal implant surgery are shown. There is evidence of increased tracer uptake in the transplanted areas, indicating the presence of surviving dopaminergic grafts at 6 and 12 months after surgery.

\section{DISCUSSION}

The use of neural transplantation to treat neurological conditions such as PD has the potential to be an important therapeutic strategy in the near future. There is strong evidence of long-term survival of 
transplanted dopaminergic neurons,[10] and clinical results are promising.[7,23] Transplantation in patients with Huntington's disease has also been reported,[9] and porcine xenografts are being studied in clinical trials. $[3,8,20]$ A great deal of experimental work in animals is being conducted using novel cell types as an alternative source of human fetal tissue for neural transplantation. This research may expand the use of reconstructive strategies in the future.[1,4,21]

We present here a simple and reliable method to deposit graft material into the brain by using a transplantation cannula and microinjector system that is easily adaptable to any stereotactic frame. We validated the two-hole design of the cannula tip in the animal experiments, which demonstrated the ability of the cannula to deliver two distinct graft deposits per injection. The design of the system allows for graft deposits to be placed no more than $3 \mathrm{~mm}$ apart from each other. This distance is close enough for the grafts to become confluent, as fiber outgrowth has been shown to extend 2 to $7 \mathrm{~mm}$ into the host tissue in human transplants.[10] In rats in which grafts were implanted, the cannula tract facilitated the connection of the two graft deposits. Proper distribution of graft deposits to facilitate confluency in all three dimensions may improve host reinnervation and clinical outcome.[5,6]

Implantation-related trauma is known to be detrimental to graft survival, $[15,16]$ and the results of our animal experiments showed excellent graft survival and no significant trauma to the transplanted rat striatum, which is an indication of the atraumatic nature of the cannula design. This observation in the experimental model correlates well with the absence of hemorrhage or tissue damage observed on the 24-hour postoperative MR images obtained in our eight patients. There was also an increase in fluorodopa uptake demonstrated on PET scanning posttransplantation. At present, the only valid method to assess graft survival in vivo is to use PET studies to measure fluorodopa. Fluorodopa is an analog of levodopa, which is taken over the blood-brain barrier, decarboxylated, and stored in the nigrostriatal dopaminergic terminals. Correlation of graft survival and fluorodopa PET scans has been made by a postmortem examination of a case in which fetal VM tissue was implanted 18 months before the patient died of causes unrelated to the transplant procedure.[10]

The cannula was designed to optimize host reinnervation by maximizing the number of deposits per pass. Increasing the density of reinnervation per pass may lead to a reduction in the number of passes through the brain and thereby reduce the chance of hemorrhagic complications. The cannula has been used with cell suspensions that are not completely dissociated and contain "chunks" of fetal VM, and no problem has arisen with the aspiration or delivery of this "chunky" cell preparation. Delivery of solid "cores" of fetal VM by using a "double-cannula system"[2] has been previously described in the literature.

In summary, we present our experience with a simple, safe, and reliable neural transplantation delivery system. As neural transplantation evolves and the clinical efficacy of this strategy for the treatment of neurological conditions is established, the ability to deliver viable grafts with minimal procedure-related trauma may play an important role in neurosurgery.

\section{Acknowledgments}

The authors wish to acknowledge Mr. Damaso Sadi for his technical support and Ms. Tanya Acorn for her assistance in the preparation of this manuscript.

\section{Disclaimer}

The Queen Elizabeth II Health Sciences Centre has filed a Canadian Patent Application for the neural transplantation cannula and microinjector system described in this report and has listed Dr. Ivar Mendez 
as the inventor. The Queen Elizabeth II Health Sciences Center has a financial interest in the device.

\section{References}

1. Borlongan CV, Tajima Y, Trojanowski JQ, et al: Transplantation of cryopreserved human embryonal carcinoma-derived neurons (NT2N cells) promotes functional recovery in ischemic rats. Exp Neurol 149:310-321, 1998

2. Breeze RE, Wells TH Jr, Freed CR: Implantation of fetal tissue for the management of Parkinson's disease: a technical note. Neurosurgery 36:1044-1048, 1995

3. Deacon T, Schumacher J, Dinsmore J, et al: Histological evidence of fetal pig neural cell survival after transplantation into a patient with Parkinson's disease. Nat Med 3:350-353, 1997

4. Fitoussi N, Sotnik-Barkai I, Tornatore C, et al: Dopamine turnover and metabolism in the striatum of parkinsonian rats grafted with genetically-modified human astrocytes. Neuroscience 85:405-413, 1998

5. Freed CR, Breeze RE, Rosenberg NL, et al: Survival of implanted fetal dopamine cells and improvement 12 to 46 months after transplantation for Parkinson's disease. N Engl J Med 327:1549-1555, 1992

6. Freeman TB, Olanow CW, Hauser RA, et al: Bilateral fetal nigral transplantation into the postcommissural putamen in Parkinson's disease. Ann Neurol 38:379-388, 1995

7. Hauser RA, Freeman TB, Snow BJ, et al: Long-term evaluation of bilateral fetal nigral transplantation in Parkinson disease. Arch Neurol 56:179-187, 1999

8. Isacson O, Deacon TW, Pakzaban P, et al: Transplanted xenogeneic neural cells in neurodegenerative disease models exhibit remarkable axonal target specificity and distinct growth patterns of glial and axonal fibers. Nat Med 1:1189-1194, 1995

9. Kopyov OV, Jacques S, Kurth M, et al: Fetal transplantation for Huntington's disease: clinical studies, in Freeman TB, Widner H (eds): Cell Transplantation for Neurological Disorders. Totowa, NJ: Humana Press, 1998, pp 95-134

10. Kordower JH, Freeman TB, Snow BJ, et al: Neuropathological evidence of graft survival and striatal reinnervation after the transplantation of fetal mesencephalic tissue in a patient with Parkinson's disease.

N Engl J Med 332:1118-1124, 1995

11. Mehta V, Spears J, Mendez I: Neural transplantation in Parkinson's disease. Can J Neurol Sci 24:292-301, 1997

12. Mendez I, Hong M: Reconstruction of the striato-nigro-striatal circuitry by simultaneous double dopaminergic grafts: a tracer study using fluorogold and horseradish peroxidase. Brain Res 778:194-205, 1997

13. Mendez I, Sadi D, Hong M: Reconstruction of the nigrostriatal pathway by simultaneous intrastriatal and intranigral dopaminergic transplants. J Neurosci 16 (22):7216-7227, 1996

14. Nikkah G, Cunningham MG, Cenci MA, et al: Dopaminergic microtransplants into the substantia nigra of neonatal rats with bilateral 6-OHDA lesions. I. Evidence for anatomical reconstruction of the 
nigrostriatal pathway. J Neurosci 15 (5):3548-3561, 1995

15. Nikkah G, Cunningham MG, Jödicke A, et al: Improved graft survival and striatal reinnervation by microtransplantation of fetal nigral cell suspensions in the rat Parkinson model. Brain Res 633:133-143, 1994

16. Nikkhah G, Olsson M, Eberhard J, et al: A microtransplantation approach for cell suspension grafting in the rat Parkinson model: a detailed account of the methodology. Neurology 63:57-72, 1994

17. Olanow CW, Kordower JH, Freeman TB: Fetal nigral transplantation as a therapy for Parkinson's disease. Trends Neurosci 19:102-109, 1996

18. Rehncrona S: A critical review of the current status and possible developments in brain transplantation. Adv Tech Stand Neurosurg 23:3-46, 1997

19. Remy P, Samson Y, Hantraye P, et al: Clinical correlates of [ $\left.{ }^{18} \mathrm{~F}\right]$ fluorodopa uptake in five grafted parkinsonian patients. Ann Neurol 38:580-588, 1995

20. Schumacher JM, Isacson O: Neuronal xenotransplantation in Parkinson's disease. Nat Med 3:474-475, 1997 (Letter)

21. Svendsen CN, Clarke DJ, Rosser AE, et al: Survival and differentiation of rat and human epidermal growth factor-responsive precursor cells following grafting into the lesioned adult nervous system. Exp Neurol 137:376-388, 1996

22. Tabbal S, Fahn S, Frucht S: Fetal tissue transplantation in Parkinson's disease. Curr Opin Neurol 11:341-349, 1998

23. Wenning GK, Odin P, Morrish P, et al: Short- and long-term survival and function of unilateral intrastriatal dopaminergic grafts in Parkinson's disease. Ann Neurol 42:95-107, 1997

Manuscript received July 21, 1999.

Accepted in final form August 19, 1999.

Address reprint requests to: Ivar Mendez, M.D., Ph.D., Division of Neurosurgery, Queen Elizabeth II Health Sciences Centre, New Halifax Infirmary, 1796 Summer Street, Room \#3806, Halifax, Nova Scotia, Canada B3H 3A7. email: mendez@is.dal.ca. 